\title{
A stabilized tunable dual wavelength erbium-doped fiber laser with equal output power
}

\begin{abstract}
Tunable dual-wavelength erbium-doped fiber laser with good stability is experimentally demonstrated. Output power of as high as $+5.26 \mathrm{dBm}$ is obtained in dual ring configuration. Both laser output can be tuned to as closed as $0.8 \mathrm{~nm}$ spacing between each other, with tuning range of $31.33 \mathrm{~nm}$. The stability of both outputs was tested that results in less than $\pm 0.8 \mathrm{~dB}$ and $\pm 0.01 \mathrm{~nm}$ in power and wavelength fluctuation, respectively.
\end{abstract}

Keyword: Dual-ring; Dual-wavelength; Erbium doped fiber laser; Good stability; Laser output; Output power; Tuning ranges; Wavelength fluctuations 